This is the final peer-reviewed accepted manuscript of:

D. Pani, A. Dessì, J. F. Saenz-Cogollo, G. Barabino, B. Fraboni and A. Bonfiglio, Fully Textile, PEDOT: PSS Based Electrodes for Wearable ECG Monitoring Systems, in IEEE Transactions on Biomedical Engineering, vol. 63, no. 3, pp. 540-549, March 2016.

The final published version is available at: http://dx.doi.org/10.1109/TBME.2015.2465936

Rights / License:

The terms and conditions for the reuse of this version of the manuscript are specified in the publishing policy. For all terms of use and more information see the publisher's website. 


\title{
Fully textile, PEDOT:PSS based electrodes for wearable ECG monitoring systems
}

\author{
Danilo Pani*, Member, IEEE, Alessia Dessì, Member, IEEE, Jose F. Saenz-Cogollo, Gianluca \\ Barabino, Beatrice Fraboni and Annalisa Bonfiglio, Member, IEEE
}

\begin{abstract}
Goal: to evaluate a novel kind of textile electrodes based on woven fabrics treated with PEDOT:PSS, through an easy fabrication process, testing these electrodes for biopotential recordings. Methods: Fabrication is based on raw fabric soaking in PEDOT:PSS using a second dopant, squeezing and annealing. The electrodes have been tested on human volunteers, in terms of both skin contact impedance and quality of the ECG signals recorded at rest and during physical activity (power spectral density, baseline wandering, QRS detectability, and broadband noise). Results: The electrodes are able to operate in both wet and dry conditions. Dry electrodes are more prone to noise artifacts, especially during physical exercise and mainly due to the unstable contact between the electrode and the skin. Wet (saline) electrodes present a stable and reproducible behavior, which is comparable or better than that of traditional disposable gelled $\mathrm{Ag} / \mathrm{AgCl}$ electrodes. Conclusion: the achieved results reveal the capability of this kind of electrodes to work without the electrolyte, providing a valuable interface with the skin, due to mixed electronic and ionic conductivity of PEDOT:PSS. These electrodes can be effectively used for acquiring ECG signals. Significance: Textile electrodes based on PEDOT:PSS represent an important milestone in wearable monitoring, as they present an easy and reproducible fabrication process, very good performance in wet and dry (at rest) conditions and a superior level of comfort with respect to textile electrodes proposed so far. This paves the way to their integration into smart garments.
\end{abstract}

Index Terms-Biomedical electrodes, Electrocardiography, PEDOT:PSS, Textile technology, Textile Electrodes

\section{INTRODUCTION}

$\mathrm{E}$ ARLY detection of diseases and health monitoring are primary goals of today's healthcare systems [1]. Long-term monitoring of electrocardiographic (ECG) signals can provide enhanced diagnostics at a low cost, by capturing intermittent episodes in arrhythmias and ischemia [2]. Commercial dynamic ECG recorders (Holter), progressively more lightweight and portable, are paving the way to a new generation of unobtrusive diagnostic/monitoring systems. Systems like CardioLeaf ULTRA by Clearbridge, or ZIO by iRhythm Technologies, mix the traditional electrodes technologies with wearable microelectronic implementations for long-term ECG

Submitted February 06, 2015; revised June 07, 2015; accepted August 2, 2015. The work of A. Dessì was supported by Sardinia Regional Government (POR Sardegna FSE Operational Programme of the Autonomous Region of Sardinia, European Social Fund 2007-2013-Axis IV Human Resources, Objective 1.3, Line of Activity 1.3.1). monitoring. However, a step further of the research is needed in order to overcome the limits of the current sensors, embedding them in the garments [3] as textile electrodes for intelligent biomedical clothing [4].

The application of textile electrodes embedded into smart garments for ECG monitoring ranges from elderly monitoring (heart failure prevention) [5] to tele-health [6], including sport or extreme conditions [6], infants monitoring [7] and clinical use [8] [9]. The same technology has also been extended to nongarment textiles, like those on household furniture, for similar purposes [9] [10], or to produce headbands to record other electrophysiological signals such as EMG [11] and EEG [12] [13]. Textile electrodes are expected to overcome the main limits of the widely used $\mathrm{Ag} / \mathrm{AgCl}$ disposable electrodes, which require a gel electrolyte to properly work. This gel (along with the electrode adhesive), when applied for a long time, can stimulate the skin, causing rash or reaction [14]. Moreover, gel drying causes increased impedance, which in turn means more noise and artifacts [15]. Dry textile electrodes can overcome these problems, with the drawback of a higher contact impedance [16], due to the material but also to the irregular electrode surface [17]. Most dry textile electrodes reported in the recent literature exhibit high skin contact impedance, which either limits their use when very small signals must be recorded [12] or poses doubts about the achievable signal quality [18] [19], even though some examples of clinically acceptable results have been recently provided [8]. These textile electrodes are usually based on fine metal wires, or metal coated yarns, woven or stitched with conventional textile fibers. Different materials and fabrication methods have been presented so far [20]. With the development of conductive polymers, it became possible to transform non-conductive textile fibers into flexible and reliable conductors [21]. Among the different available conducting polymers, poly-3,4-ethylenedioxythiophene doped with poly(styrene sulfonate) (PEDOT:PSS) is one of the most used. It has attracted wide attention as electrode material in several applications, due to its excellent characteristics such as low band gap and superior electrochemical and thermal stability. Due to its biocompatibility, it has been used to create electrodes for recording extracellular potentials both in vitro and in vivo [22]. PEDOT:PSS treated fabrics, employed for

D. Pani*, A. Dessì, G. Barabino, Jose F. Saenz-Cogollo and A. Bonfiglio are with the Dept. of Electrical and Electronic Engineering, University of Cagliari, Italy (correspondence e-mail: pani@diee.unica.it, annalisa@diee.unica.it).

B. Fraboni is with the Dept. of Physics and Astronomy, University of Bologna, Italy. 
electrodes fabrication, have an invaluable advantage compared to other conductive textiles: they show a decreased electrochemical impedance mismatch between tissue and electrode, thus avoiding the need for conductive gels. This is due to their mixed electronic and ionic conductivity and high ionic mobility. However, so far, there have been a few attempts to use PEDOT:PSS for making textile electrodes relying either on a rigid support [23] or on a semi-rigid $\mathrm{Ag} / \mathrm{AgCl}$ wire [24].

In this paper, we present a novel approach for the fabrication of bio-potential electrodes, based on the treatment of a standard textile fabric with a highly conductive PEDOT:PSS solution. The performance of the proposed electrodes has been compared with that of disposable gelled $\mathrm{Ag} / \mathrm{AgCl}$ electrodes in ECG recordings on human volunteers. The quality of the recorded signals, under static (rest) and dynamic (exercise) conditions, has also been assessed. A small-sized custom Holter monitor has been developed to independently record signals from pairs of different electrodes with known analog processing. The achieved results are very promising, opening the door to the possibility of developing smart garments for unobtrusive ECG monitoring, as well as to the extension of their use for recording other biopotentials.

\section{MATERIALS AND METHODS}

\section{A. Electrodes Fabrication}

Textile electrodes are made by treating conventional fabrics with a highly conductive solution of PEDOT:PSS. It is widely known that, in order to achieve reasonable conductivity values of PEDOT:PSS, a second dopant should be used. The conductivity can be enhanced from one to three orders of magnitude by the addition of poly-alcohols (alcohols with more than two $\mathrm{OH}$ groups on each molecule, e.g. ethylene glycol, glycerol, sorbitol) or high-dielectric solvents (dimethyl sulfoxide, N-N dimethylformamide, tetrahydrofuran) [15] [25] [26]. For the preparation of the conductive fabrics, we chose to test the effect of sorbitol, ethylene glycol and glycerol since they have reported the highest conductivities [15] [27] [28]. The screened chemicals were mixed at different proportions with the PEDOT:PSS dispersion Clevios PH 500 (Heraeus Clevios - Germany) with a magnetic stirrer until a homogeneous solution was obtained. Woven cotton $(250 \mu \mathrm{m}$ thick $)$ and polyester fabrics (400 $\mu \mathrm{m}$ thick) were used as received, and immersed for at least $48 \mathrm{~h}$ at room temperature in the polymer solution. Fabrics were then removed from the PEDOT solution and squeezed between metallic rolling cylinders to remove the solution in excess. Samples were then annealed at $180{ }^{\circ} \mathrm{C}$ for 3 minutes, roughly the time needed for both water and second dopant to evaporate in order to avoid deterioration of the fabric mechanical properties.

Dried fabrics were cut in $35 \mathrm{~mm} \times 65 \mathrm{~mm}$ pieces and the surface resistance measured. In order to evaluate the properties of the conductive fabrics as surface bio-potentials electrodes, without any disturbance due to other materials getting in touch with the skin, the conductive fabric was sewed to a nonconductive $35 \mathrm{~mm} \times 35 \mathrm{~mm}$ layered structure of foam $(4 \mathrm{~mm})$ and polyester, using simple cotton yarn. In order to obtain

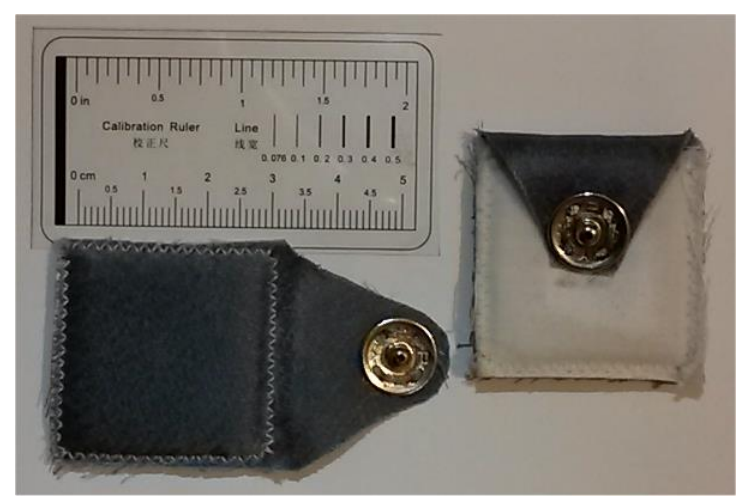

Fig. 1. The prototypical PEDOT:PSS textile electrode.

reproducible samples, all sewing was performed following an automatic procedure developed with an electronic embroidering machine (Brother Innovi's 955). After sewing, the active area of electrodes is reduced to $30 \mathrm{~mm} \times 30 \mathrm{~mm}$. The size of the electrodes, which is acceptable for ECG monitoring, has been chosen to ensure a good reproducibility of the custom fabrication process. The introduction of foam layers allows improving the contact between the electrode and the skin by ensuring a uniform pressure, which is especially beneficial in case of dry electrodes. In fact, some authors associate the baseline drift, which is a typical artifact in signals acquired by textile electrodes, to a variation in the contact pressure, which can be reduced by a soft layer of foam [29]. The presence of the foam also allows keeping the electrode wet (physiologically or artificially) in order to reduce the contact impedance [12].

The exceeding $20 \mathrm{~mm} \times 30 \mathrm{~mm}$ of conductive fabric on one side was used to attach a metallic snap fastener, as shown in Fig. 1, which has a termination similar to the commercial disposable electrodes, allowing a direct connection with standard patient cables. The snap fastener was fixed to the fabric with a silver conductive paste and then secured by sewing it with a silver-coated yarn (Shieldex $117 / 12 \times 2$ ply, Statex Germany). In such a way, the snap fastener remains in a tail of the fabric that can be flipped over the rear of the electrode not getting in touch with the skin. It should be noted that, similarly to other relevant works in the field [30], the focus of this paper is on the evaluation of this novel kind of textile electrode rather than on its integration in a smart garment. However, given the extreme versatility of the fabrication technique, an integration of the electrode in a garment could be obtained by selectively treating pre-defined portions of the garment with a similar procedure.

The choice of woven cotton and polyester fabrics was motivated by the idea of having common textile materials instead of rare and expensive ones. Notably, the proposed approach can be applied to any kind of textile material able to absorb and retain the conductive polymer. Furthermore, we chose flexible but unstretchable fabrics in order to reduce the effect of the stretch on the electrode-skin contact impedance [17]. In fact, flexibility and stretchability can confer to conductive textiles the ability to act as strain gauges, changing their impedance as a function of the stretch level [31]. Clearly, this feature is not desirable for an electrode, which should maintain its impedance constant despite the occurrence of 
mechanical deformations.

\section{B. Experimental Setup}

Several experimental strategies were adopted in order to evaluate the different aspects of the textile electrodes. In the absence of any guideline for testing the textile electrodes, available, on the contrary, for disposable gelled ones [15], we performed the analyses looking at commonly used meaningful figures of merit in the field.

In order to evaluate the conductivity of treated fabrics and compare the different materials and treatments, we used a Source Meter 2336/2612 (Keithley Instruments, Ohio - USA) in a 4-wire configuration. A current of $1.0 \mathrm{~mA}$ was applied through $40 \mathrm{~mm}$ long flat clamps separated $40 \mathrm{~mm}$ between them. The voltage, measured at a constant current between pin electrodes separated $28 \mathrm{~mm}$ over the fabric, was used to estimate the fabric conductivity. We calculated the conductivity of the fabric using the equation that relates the resistance and the conductivity of a flat sheet of solid material. Since a fabric is not a solid with a defined thickness, such calculation is only an approximation, good enough for the purposes of this evaluation.

The skin contact impedance of 20 textile electrodes was measured on a single subject (hereafter called subject 1 ) in order to evaluate the variability of the electrodes characteristics. Two types of impedance measurements were made: one at $10 \mathrm{~Hz}$ using the FDA cleared Prep-Check impedance meter (General Devices) and one at other 9 different frequencies ranging from $20 \mathrm{~Hz}$ to $250 \mathrm{~Hz}$ using a high precision impedance meter (LCR Meter Agilent 4282A). The lower frequency limit is imposed by the instrument, whereas the upper one is meaningful in the light of the international recommendations for the interpretation of clinical rest ECG in adults and infants [42]. To improve reproducibility, a light skin preparation (with Everi Cream by Spes Medica) has been carried out in the area where the test electrode had to be placed (i.e. in the right hemithorax, in the space between the anterior axillary and mid-axillary lines). Noticeably, a mild skin preparation has a low impact on the dry electrode-skin contact impedance [16], but it is able to improve the homogeneity within the experimental group, for both textile and $\mathrm{Ag} / \mathrm{AgCl}$ electrodes, achieving comparable starting conditions without affecting the intrinsic inter-subject differences. The electrodes have been applied under an elastic belt, $47 \mathrm{~mm}$ large, positioned just below the chest muscles [16] [32]. The use of chest belt allows keeping the electrode in place ensuring a good electrical contact with the skin, constantly over time, during the impedance measurement [33].

Two commercial $\mathrm{Ag} / \mathrm{AgCl}$ electrodes (FIAB F9079, foam with solid hydrogel) have been applied just below the belt, 10 $\mathrm{mm}$ spaced, in the same anatomical area. For them, a deep skin preparation consisting of gentle scrub with Everi Cream followed by a harder mechanical scrub (with a thin plastic scraper and Neurgel by Spes Medica) has been performed. Neurgel has been left in place to improve the conductivity of the solid hydrogel of the commercial electrodes. The skin contact impedance at $10 \mathrm{~Hz}$ for the $\mathrm{Ag} / \mathrm{AgCl}$ electrode undergoing this hard skin preparation, measured as depicted on the right part of Fig. 2 between terminals 2 and R, and halving the value read on the Prep-Check impedance meter, was about

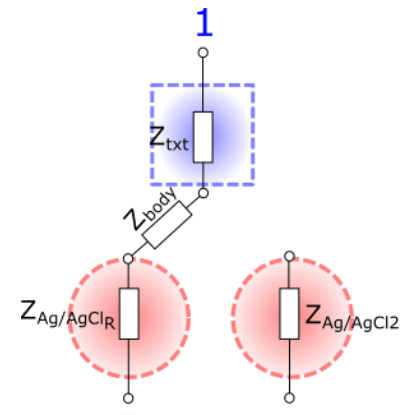

$\mathrm{R}$
2
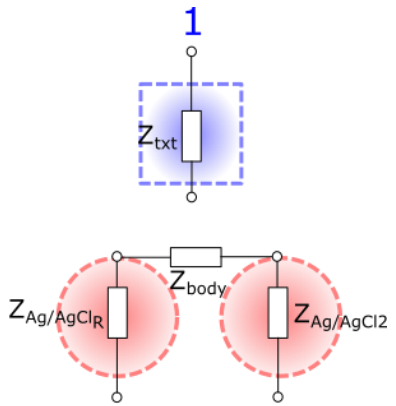

$\mathrm{R}$

2
Fig. 2. Equivalent electrical diagrams of the acquisition setup used for the impedance measurement with the FDA approved Prep-Check meter. The textile electrode is depicted as a blue square, the $\mathrm{Ag} / \mathrm{AgCl}$ electrodes as red circles. It should be noted that $\mathrm{Z}_{\text {body }}$ is negligible compared to the electrodeskin contact impedance in both cases.

$9 \mathrm{k} \Omega$, including one half of the body impedance contribution. The impedance measurement at $10 \mathrm{~Hz}$ has been carried out between the textile electrode and one of the $\mathrm{Ag} / \mathrm{AgCl}$ electrodes, i.e. between terminal 1 and $\mathrm{R}$ in the left part of Fig. 2 , so that the measured value includes both the skin-textile electrode and the skin- $\mathrm{Ag} / \mathrm{AgCl}$ electrode contact impedance contributions, plus the negligible body impedance. Such an inhomogeneous setup, common to other works in the field [34], has been chosen to avoid saturation of the Prep-Check impedance meter, exhibiting a saturation value of $200 \mathrm{k} \Omega$. Moreover, this instrument provides only the modulus of the impedance, avoiding any a posteriori correction.

The same setup was then used for the impedance evaluation at the other frequencies, from 20 to $250 \mathrm{~Hz}$, exploiting the LCR meter, keeping the injected sinusoidal current below $1 \mu \mathrm{A}$. In this case, the two $\mathrm{Ag} / \mathrm{AgCl}$ electrodes have been used to measure the skin contact impedance on the same points, obtaining a reference complex impedance to be subtracted from the measured one: This leads a good estimate of the skin-textile electrode contact impedance. Such a measurement has been performed only on subject 1 .

In order to reduce the noise potentially affecting these impedance measurements, regardless the adopted instrument, they have been performed in an anechoic chamber with filtered mains and lamps with reduced electromagnetic emission at the low frequencies. The presence of an isolation transformer also represents a safety measure for the impedance evaluation with the LCR meter on subject 1. The temperature of the room has been kept at $25{ }^{\circ} \mathrm{C}$ during all the measurements, in order to reduce the influence of this parameter on the skin conductivity. A delay of 5 minutes between electrodes positioning and impedance measurement has been provided to ensure the electrode stabilization [15] [19].

Ten healthy volunteers of the research group (aged 29 \pm 4 , BMI $22.3 \pm 2.0$ ) were enrolled in the study to evaluate both electrodeskin contact impedance and ECG signals quality. The study has been performed following the principles outlined in the Helsinki Declaration of 1975, as revised in 2000; all the volunteers gave their informed consent to the measurements, which lasted from 20 to 30 minutes per subject. The acquisition protocol for impedance measurement was the same described above, including the skin preparation procedure, but only the FDA cleared device at $10 \mathrm{~Hz}$ has been used to measure the total impedance between textile and $\mathrm{Ag} / \mathrm{AgCl}$ electrodes. 
For ECG signal acquisition, rather than choosing convenient positions to reduce the artifacts, e.g. as in [8] [16], we opted for the single lead standard Holter placement (second lead for 7electrode configuration). In this configuration, the negative electrode is placed close to the right clavicle (lateral to the midclavicular line) and the positive one is placed close to the $6^{\text {th }}$ intercostal space on the anterior axillary line. Two couples of electrodes have been positioned, the textile ones (fixed by means of a TNT adhesive surgical tape) above the line connecting said points, the $\mathrm{Ag} / \mathrm{AgCl}$ ones below the same line, so that the homologous electrodes lay on the perpendicular to the lead direction. As ground electrode, the same $\mathrm{Ag} / \mathrm{AgCl}$ electrode used for the impedance measurement was chosen. The obtained lead is approximately oriented along the cardiac axis, where the highest signals can be measured [16]. The choice of a standard placement has the intrinsic advantage of conveying meaningful information for a visual inspection by a cardiologist.

The experimental protocol was designed to include both rest (subject sitting, no physical movements, normal breathing, 60 seconds of acquisition) and dynamic conditions (about 30 seconds for each different condition):

- deep breathing (subject sitting, six deep respiratory acts)

- stairs climbing up and down, following a $1 \mathrm{~Hz}$ pace (one stair-step per second)

- $\quad$ step exercise, following a $2 \mathrm{~Hz}$ pace (every half second a movement up or down the step)

Despite the impedance measurements have been performed in the closed anechoic chamber, as motivated before, ECG signals have been acquired in normal lab rooms and other generic departmental structures, to avoid any attenuation of the typical electromagnetic interferences present in common environment. Both dry and wet (two drops of saline solution, sodium chloride $0.9 \%$, about $0.10 \mathrm{ml}$ ) conditions were tested. Saline was chosen because it is a liquid with a composition similar to the human sweat, normally acting as a weak electrolyte [12], and to ensure reproducibility of the achieved results compared to the simple tap water exploited in other works [15].

A 48-hour test on a subject, with impedance measurement performed at $10 \mathrm{~Hz}$ exploiting the portable Prep-Check meter every hour during the daytime and keeping the electrodes in place during the night, has been carried out to evaluate the durability of the electrodes and the drying time. The measurements have been performed by the subject itself, in different locations, according to his activity.

Throughout the results section, box and whiskers plots have been used for the pictorial representation of samples distributions, since the largest part of them are not symmetric. In such figures, the median is highlighted, the box defines the $50 \%$ of the samples between the first and third quartile, and the wishers range from the minimum to the maximum value, excluding the outliers (represented with crosses). The outliers are defined as data larger than $q_{3}+1.5\left(q_{3}-q_{1}\right)$ or smaller than $q_{1}-1.5\left(q_{3}-q_{1}\right)$, where $q_{1}$ and $q_{3}$ are the $25^{\text {th }}$ and $75^{\text {th }}$ percentiles, respectively, corresponding to approximately $\pm 2.7 \sigma$ and 99.3 coverage, if the data are normally distributed.

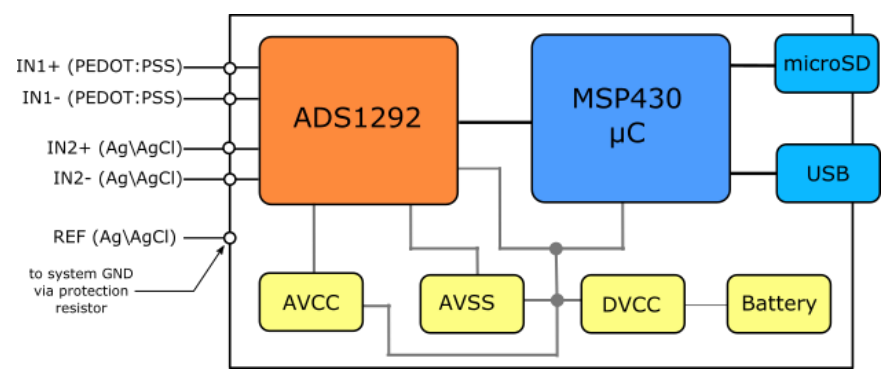

Fig. 3. Block diagram of the recording unit and its connection to the subject during the performed tests. The supply voltages for both the analog section AVCC $(+2.5 \mathrm{~V})$, AVSS $(-2.5 \mathrm{~V})$ and the digital one DVCC $(+3.3 \mathrm{~V})$ are derived from a single power source (Battery).

\section{1) Recording Unit}

In order to provide a fair comparison of the ECG signals acquired by means of different electrodes, a dedicated wearable bio-potentials recording system has been designed. Compared to clinical ECG recorders, it allows acquiring two ECG channels simultaneously and independently, with minimal and known signal conditioning before $\mathrm{A} / \mathrm{D}$ conversion in order to better evaluate quality differences among signals in various conditions. The developed recording unit is based on the ADS1292 monolithic analog front-end, specifically designed for ECG applications, with programmable input gain and $1 \mathrm{G} \Omega$ input impedance. It features a 24-bit analog to digital converter (ADC), composed of two $\Sigma \Delta$ converters, which operate in parallel to obtain a simultaneous acquisition. This architecture relaxes the constraints for the input anti-aliasing filter thanks to the decimation filter at the ADC output. In fact, only an adequate attenuation at multiples of the modulator input sampling frequency is needed. A sampling frequency of 512 $\mathrm{Hz}$, derived from a modulator frequency of $128 \mathrm{kHz}$, has been chosen. A value of $4.7 \mathrm{nF}$ of the external capacitor included in the input amplifier of each channel sets the cut-off frequency of the low-pass input filter to $8.4 \mathrm{kHz}$, which leads to a minimal ECG signal distortion, according to the device specifications (by increasing the capacitance value also the total harmonic distortion degrades). An electromagnetic interference (EMI) filter precedes the input stages with a cut-off frequency set at 3 MHz.

Devices of this family have been already used in the past for the tests on textile electrodes, coupled with a National Instruments digital acquisition card [31]. In the developed wearable unit, the analog front-end is controlled by a MSP430F5515 microcontroller via an SPI port. This has been used to configure it, read the data whenever available and write them to a microSD card. The system is interfaced to the outer world via an USB port, which serves primarily as programming interface. The same port can be used also to stream data to a PC, but this feature has not been exploited in this test. The device, which weighs only $102 \mathrm{~g}$, is supplied by a single $4.2 \mathrm{~V}$ rechargeable Li-Ion battery.

A reference electrode connected to the subject can be internally tied to a right-leg-drive circuitry or simply to the system reference via a protection resistor. The latter configuration has been chosen here, due to its passive nature and the limited benefit of the active drive revealed by our tests [7]. The measurements have been carried out using unshielded cables (MLA0315, ADInstruments Inc.) connected to the safelead recessed connectors of the recording unit. A block diagram 
of the recording unit in the setup exploited for the ECG measurements is presented in Fig. 3.

\section{Performance Indexes and Signal Processing}

The measurements, aiming at assessing the quality of the signals, require data post-processing to extract valuable figures of merit. The adopted techniques are presented here.

To evaluate the broadband noise in absence of any physiological signal of interest, the rms around the isoelectric line has been adopted. An estimation of the electrical noise could also be obtained through the acquisition of a signal from a silent part of the body, with respect to the ECG, (e.g., where the impedance measurement is performed). However, by processing real ECG signals it is possible to include all the conditions that are present in the real ECG measurement the rms value is referred to (impedance, noise coupling, etc.), leading to an index coherent with the other parameters extracted from the signal. Such an approach, with a different processing technique to isolate the noise, has been also used in [15] and in other works.

At first, the very low-frequency components are removed from the signal. This is needed since one of the main problems of dry electrodes in the literature is the presence of baseline wandering artifacts in the signal. Different interpretations have been given to this phenomenon, partly attributed to the material characteristics [15] and partly to contact stability (pressure) [29], which in turn depends on the high electrode-skin contact impedance. Such a narrowband noise is quite intense, and it could mask other signal components in the spectrum. For this reason, it is usually removed also for PSD analysis. In this work, we removed the baseline wandering interference by using a non-linear filter [36], avoiding traditional linear filtering to limit spectral distortion. The signal baseline is estimated and saved for further use, then subtracted from the original signal. Similarly, in [12], a linear detrending procedure is used to remove the polarization trend in EEG recording but this approach is not adequate when the low-frequency artifact is not monotonic, as for the ECG signals.

After baseline cancelling, a wavelet-based ECG delineator [37] has been used to extract the segments of the signals between the end of a $\mathrm{T}$ wave and the onset of the next $\mathrm{P}$ wave. These segments have been concatenated, to isolate the parts related to the isoelectric line only. This allows estimating the broadband noise level without the interference of the main ECG waves, alternatively to residual signal analysis [15].

As said, this approach to the computation of the rms of the broadband noise does not consider the important contribution of the baseline wandering. In order to provide an estimation of the amount of low-frequency noise, it is possible to compute the rms of the centered previously extracted baseline. Centering removes the DC component, that would considerably affect the rms computation. This is equivalent to computing the standard deviation of the estimated baseline, which provides a simple informative index for the evaluation of this narrowband noise.

To check the distribution of the frequency components, on a single subject at rest, we exploited the PSD estimated with the
Welch's method (applied on a 10 seconds segment, exploiting a Hamming window of 1024 samples, equivalent to $2 \mathrm{~s}$, and 256 samples overlap). Compared to [19] and [32], a larger number of points has been chosen, limiting the plot to the most interesting part of the spectrum for ECG recordings, where the largest part of energy is represented. The frequency bins from zero to $1 \mathrm{~Hz}$ have been cleared to zero in order to emphasize the spectral components, otherwise towering over the other components producing an unreadable plot. This solution avoided any spectral modification that could derive from the application of digital filters to the signal before PSD computation, considering that our recording unit has no highpass stage (differently from others setups in the literature, where analog or digital filtering is used [30] [38]).

The quality of the signals acquired from the textile and $\mathrm{Ag} / \mathrm{AgCl}$ electrodes has also been evaluated in terms of QRS complexes detection by using the Pan-Tompkins algorithm [39]. This is a simple and widely accepted real-time algorithm, based on digital filtering with integer coefficients, generally used in portable heart rate monitoring systems. It is available in several implementations (e.g., it is embedded in the PhysioToolkit by PhysioNet [43]), and has been extensively validated on several international databases producing excellent results. Despite Pan-Tompkins is not the best algorithm, compared to other QRS detectors, by using the same detector on both the signals from textile and $\mathrm{Ag} / \mathrm{AgCl}$ electrodes, it is possible to have a fair evaluation of the electrodes quality. Such an approach is useful to evaluate several signals, especially in dynamic condition [38], providing a numerical value over the whole population.

In this case, the visual inspection and consequent manual annotation of an expert was used as the ground truth, comparing the performance of the same algorithm on the two signals for every test. Annotation is performed analyzing contemporarily all the readable channels, so that $\mathrm{R}$ peaks are annotated even though not (well) visible in a part of that specific signal, but clearly visible in the other one. No segments of a readable signal have been removed from the analysis. All the signals with no acceptable quality for any kind of QRS detection have been marked as unreadable and completely discarded. This approach is common to all the studies in the field of ECG waveform delineation and event detection [37].

\section{RESULTS}

The effect on the conductivity of the second dopant and different affinity to the PEDOT:PSS treatment of the substrate material has been measured and is presented in Table I. It can be clearly seen that polyester performs sensibly better than cotton. In preliminary tests, we also observed that treated polyester fabrics were mechanically stronger and withstand better the sewing than cotton, which becomes more fragile after treatment. Given these results, only polyester electrodes treated with glycerol were selected for impedance and ECG recordings. 
TABLE I

CONDUCTIVITY OF PEDOT:PSS COATED FABRICS; COMPARISON OF SECOND DOPANTS AND TEXTILE MATERIALS

\begin{tabular}{lccc}
\hline \hline \multirow{2}{*}{$\begin{array}{c}\text { Fabric } \\
\text { material }\end{array}$} & \multicolumn{3}{c}{ Conductivity $(\mathbf{m S} / \mathbf{c m})$} \\
\cline { 2 - 4 } & Sorbitol & Ethylene Glycol & Glycerol \\
\hline $\begin{array}{l}\text { Woven } \\
\text { Cotton }\end{array}$ & $5 \pm 1$ & $266 \pm 24$ & $424 \pm 83$ \\
\hline $\begin{array}{l}\text { Woven } \\
\text { Polyester }\end{array}$ & $220 \pm 59$ & $531 \pm 62$ & $575 \pm 70$ \\
\hline \hline
\end{tabular}

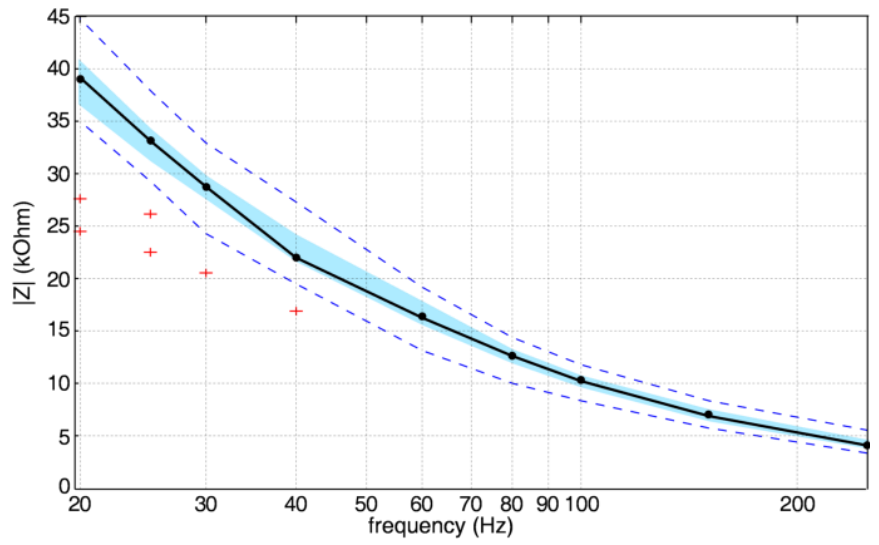

Fig. 4. Impedance trend of dry textile electrodes (20 samples), evaluated on subject 1 at different frequency values. The median impedance is the solid line, the $25^{\circ}$ percentile and the $75^{\circ}$ percentile limit the shaded area, the dashed lines span between the minimum and maximum values, not considered as outliers (which are shown as red crosses).

The results of the evaluation on subject 1 of the skin contact impedance for all the dry textile electrodes, with a sweep in frequency between $20 \mathrm{~Hz}$ and $250 \mathrm{~Hz}$, are presented in Fig. 4. The median impedance at $20 \mathrm{~Hz}$ is about $40 \mathrm{k} \Omega$, with a clear decreasing trend in frequency and a noticeable good repeatability. Such results are of the same order of magnitude of disposable gelled $\mathrm{Ag} / \mathrm{AgCl}$ electrodes.

As discussed in Section II-B, only the $10 \mathrm{~Hz}$ impedance has been investigated with the 10 enrolled volunteers, using the FDA cleared meter. The results of the impedance module are reported in Fig. 5, without any correction, so including the contribution of the skin- $\mathrm{Ag} / \mathrm{AgCl}$ electrode, which is around $9.0 \pm 2.6 \mathrm{k} \Omega$ (recalling they underwent the hardest skin preparation and gel treatment to achieve the smallest impedance). Remarkably, the impedance distribution dispersion is quite high for the dry textile electrodes, and considerably reduced for the wet ones. However, for dry electrodes, this is mainly the consequence of the inter-subject skin differences (moisture, texture, composition, etc.) rather than a characteristic of the electrode. In fact, all the electrodes tested at $10 \mathrm{~Hz}$ on the first subject present a substantially lower dispersion with similar median values, as depicted in Fig. 5.

The experimental setup also included the evaluation of the textile electrodes in ECG signal acquisition using a different electrode placement, as described in Section II, and applying for the $\mathrm{Ag} / \mathrm{AgCl}$ electrodes the same light skin treatment reserved to the textile ones.

Fig. 6 presents, for a single subject (different from subject1),

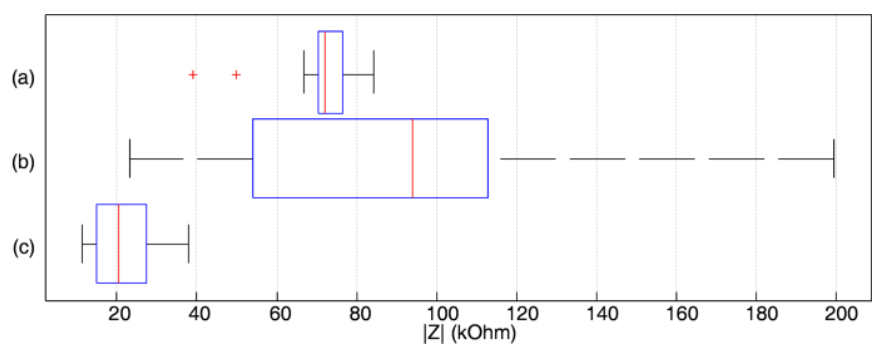

Fig. 5. Boxplot of the electrode-skin contact impedances at $10 \mathrm{~Hz}$ : (a) dry textile electrodes on subject 1, (b) dry textile electrodes on all the 10 subjects, (c) wet textile electrods on all the 10 subjects.

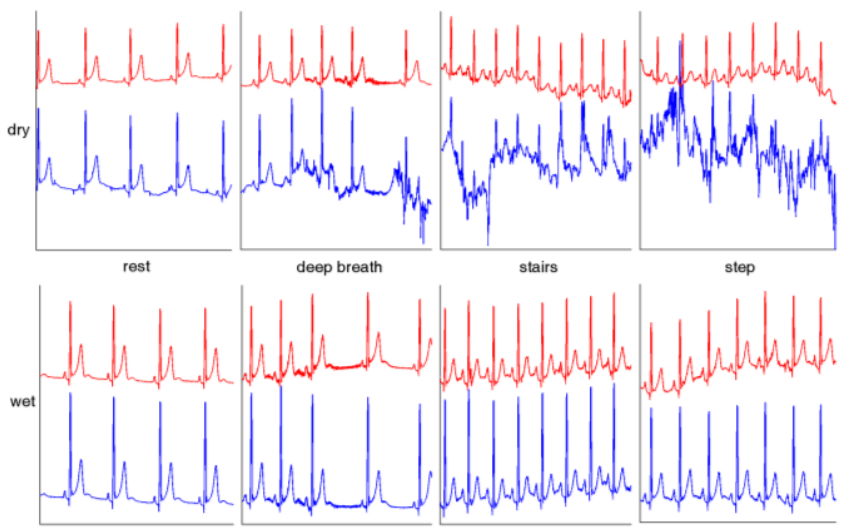

Fig. 6. Five seconds excerpts of ECG signals acquired with dry and wet electrodes (in blue, lower signals) compared to those simultaneously acquired with gelled $\mathrm{Ag} / \mathrm{AgCl}$ ones (in red, upper signals) during different activities.

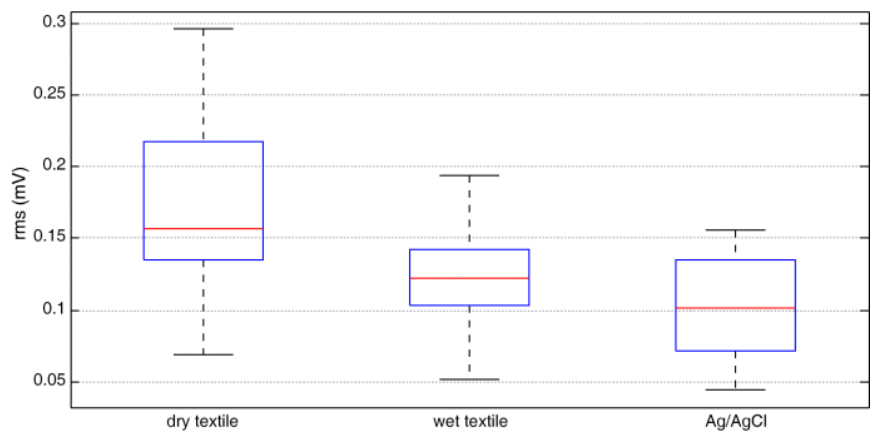

Fig. 7. rms around the isoelectric line of all the subjects for dry textile, wet textile and $\mathrm{Ag} / \mathrm{AgCl}$ electrodes for rest ECG.

5-second excerpts of ECG signal during rest and dynamic conditions. The $\mathrm{Ag} / \mathrm{AgCl}$ electrodes show better stability of the baseline compared to the dry textile electrodes, but worse than the wet ones. Performance of wet and $\mathrm{Ag} / \mathrm{AgCl}$ electrodes are similar, in general, whereas dry textile electrodes reveal higher sensitivity to high frequency noise.

For the evaluation of the signals at rest, we computed both the rms around the isoelectric line and the PSD of the signal, the former on the whole dataset of 10 subject and the latter on one subject only. Fig. 7 shows the rms distribution for dry, wet, and $\mathrm{Ag} / \mathrm{AgCl}$ electrodes at rest. The isoelectric line segments, between the end of a $\mathrm{T}$ wave and the beginning of the next $\mathrm{P}$ wave, have been extracted and appended to obtain at least a 10second signal for the rms evaluation. This corresponds to approximately 25 beats for a heart rate of about $66 \mathrm{bpm}$ (e.g., for subject 1). It is clearly visible that wet textile and $\mathrm{Ag} / \mathrm{AgCl}$ electrodes have a similar performance. Interestingly, the dispersion for the wet electrodes is even lower. 

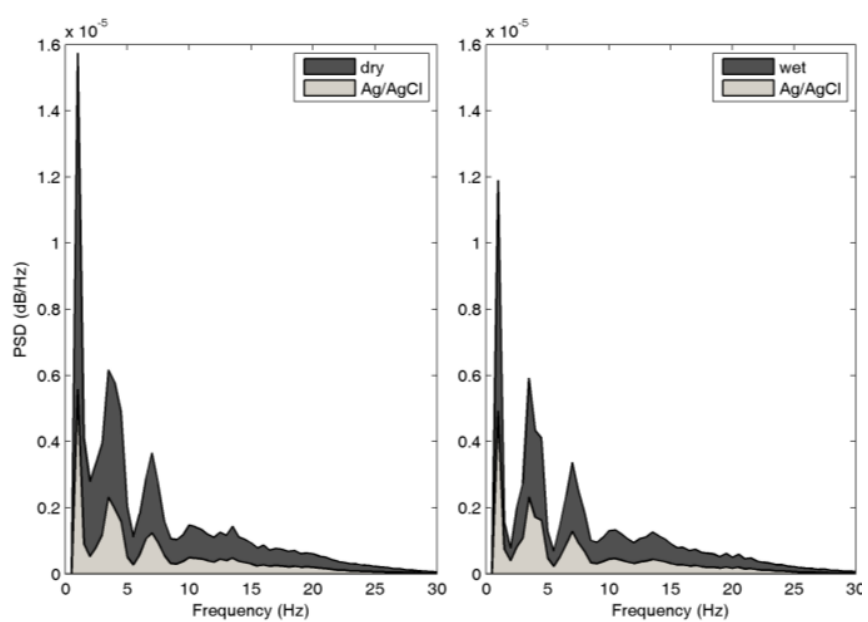

Fig. 8. PSD for $\mathrm{Ag} / \mathrm{AgCl}$ and dry/wet textile electrodes at rest.

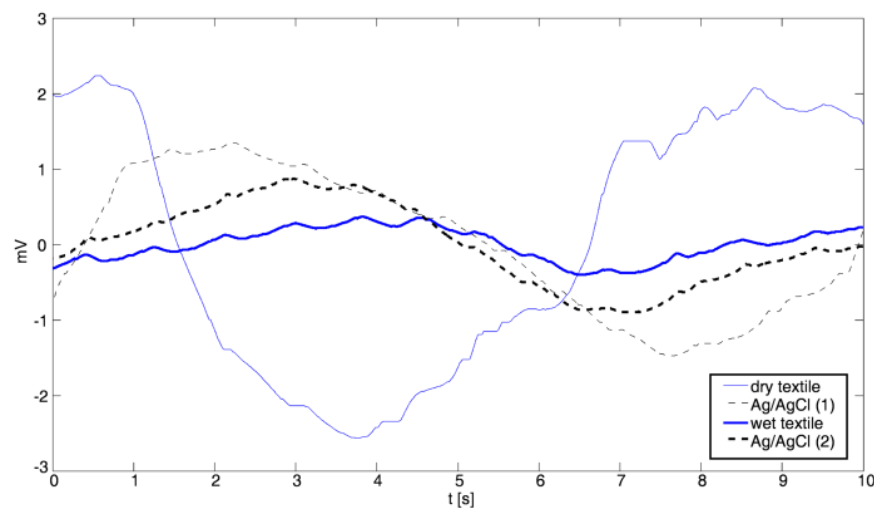

Fig. 9. Baseline wandering trend extracted through non-linear filtering during 10 seconds of deep breathing. $\mathrm{Ag} / \mathrm{AgCl}(1)$ is referred to the acquisition performed simultaneously with dry textile electrodes; $\mathrm{Ag} / \mathrm{AgCl}(2)$ to the case with wet textile electrodes.
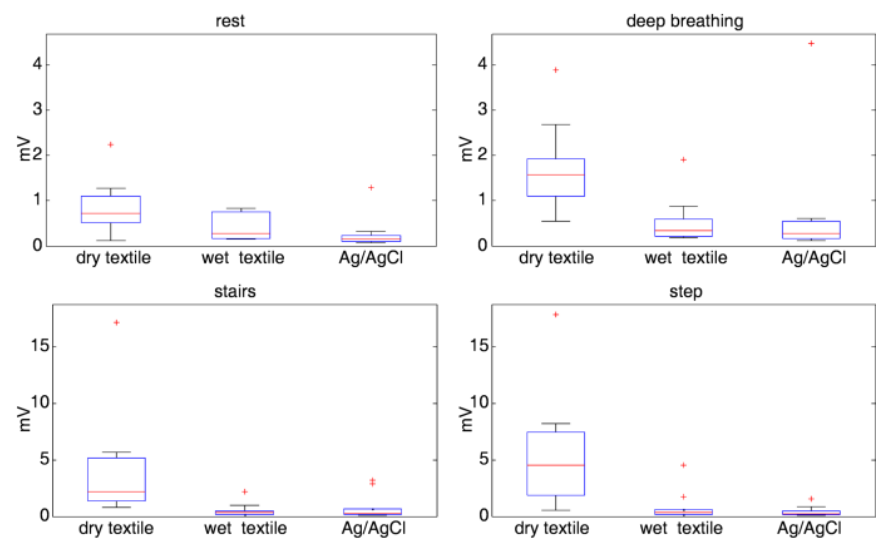

Fig. 10. Standard deviation of the baseline wandering artifact.

Fig. 8 shows the PSDs of the signals at rest acquired from subject 1, according to the method introduced in Section II.C. The spectral content for $\mathrm{Ag} / \mathrm{AgCl}$ and textile electrodes is quite similar, particularly for the peaks corresponding to the $\mathrm{T}$ wave, $\mathrm{P}$ wave and QRS complex. It is worth to note that no normalization has been computed, so the amplitude differences are due to the different signal amplitudes. Dry textile electrodes show strongest components at low frequencies than wet ones. Results are similar to those presented by [38], where a different PSD parameterization has been used along with a different positioning of the electrodes. The PSD trends of textile and
TABLE II

QRS DETECTION RESULTS IN TERMS OF SENSITIVITY S AND POSITIVE PREDICTIVITY $\mathrm{P}^{+}$OVER 10 SUBJECTS FOR DRY TEXTILE ELECTRODES. VALUES IN THE SHADED ROWS HAVE BEEN COMPUTED USING ALL THE SIGNALS READABLE IN THE AG/AGCL ELECTRODES, WHEREAS ALL THE OTHERS HAVE BEEN COMPUTED ON THE SIGNALS READABLE WITH BOTH THE ELECTRODES.

\begin{tabular}{|c|c|c|c|c|c|c|}
\hline & $\begin{array}{l}\text { Type of } \\
\text { electrode }\end{array}$ & $\begin{array}{c}\text { \# of } \\
\text { readable } \\
\text { records }\end{array}$ & $\begin{array}{c}\text { \# of } \\
\text { used } \\
\text { records }\end{array}$ & $\begin{array}{l}\text { \# of } \\
\text { beats }\end{array}$ & S\% & $\mathbf{P}^{+} \%$ \\
\hline \multirow{2}{*}{ rest } & dry textile & $10 / 10$ & \multirow{2}{*}{$10 / 10$} & \multirow{2}{*}{695} & 99,86 & 98,86 \\
\hline & $\mathrm{Ag} / \mathrm{AgCl}$ & $10 / 10$ & & & 100 & 99,71 \\
\hline \multirow{2}{*}{ breath } & dry textile & $10 / 10$ & \multirow{2}{*}{$10 / 10$} & \multirow{2}{*}{356} & 99,44 & 91,47 \\
\hline & $\mathrm{Ag} / \mathrm{AgCl}$ & $10 / 10$ & & & 100 & 99,72 \\
\hline \multirow{4}{*}{ stairs } & dry textile & $5 / 10$ & \multirow{2}{*}{$5 / 10$} & \multirow{2}{*}{151} & 93,38 & 86,50 \\
\hline & $\mathrm{Ag} / \mathrm{AgCl}$ & $8 / 10$ & & & 99,34 & 99,34 \\
\hline & dry textile & $5 / 10$ & \multirow{2}{*}{$8 / 10$} & \multirow{2}{*}{252} & 65,48 & 68,75 \\
\hline & $\mathrm{Ag} / \mathrm{AgCl}$ & $8 / 10$ & & & 99,21 & 99,60 \\
\hline \multirow{4}{*}{ step } & dry textile & $6 / 10$ & \multirow{2}{*}{$6 / 10$} & \multirow{2}{*}{331} & 87,61 & 80,78 \\
\hline & $\mathrm{Ag} / \mathrm{AgCl}$ & $10 / 10$ & & & 95,77 & 97,24 \\
\hline & dry textile & $6 / 10$ & \multirow{2}{*}{$10 / 10$} & \multirow{2}{*}{528} & 68,56 & 62,85 \\
\hline & $\mathrm{Ag} / \mathrm{AgCl}$ & $10 / 10$ & & & 97,16 & 97,71 \\
\hline
\end{tabular}

TABLE III

QRS DETECTION RESULTS IN TERMS OF SENSITIVITY S AND POSITIVE PREDICTIVITY $\mathrm{P}^{+}$OVER 10 SUBJECTS FOR WET TEXTILE ELECTRODES

\begin{tabular}{|c|c|c|c|c|c|}
\hline & $\begin{array}{l}\text { Type of } \\
\text { electrode }\end{array}$ & $\begin{array}{c}\text { \# of } \\
\text { readable } \\
\text { records }\end{array}$ & $\begin{array}{c}\text { \# of } \\
\text { beats }\end{array}$ & S\% & $\mathbf{P}^{+} \%$ \\
\hline \multirow{2}{*}{ rest } & wet textile & $10 / 10$ & \multirow{2}{*}{602} & 100 & 100 \\
\hline & $\mathrm{Ag} / \mathrm{AgCl}$ & $10 / 10$ & & 100 & 99,83 \\
\hline \multirow{2}{*}{ breath } & wet textile & $10 / 10$ & \multirow{2}{*}{334} & 100 & 100 \\
\hline & $\mathrm{Ag} / \mathrm{AgCl}$ & $10 / 10$ & & 100 & 100 \\
\hline \multirow{2}{*}{ stairs } & wet textile & $10 / 10$ & \multirow{2}{*}{329} & 99,7 & 100 \\
\hline & $\mathrm{Ag} / \mathrm{AgCl}$ & $10 / 10$ & & 99,57 & 95,25 \\
\hline \multirow{2}{*}{ step } & wet textile & $10 / 10$ & \multirow{2}{*}{492} & 98,98 & 97,79 \\
\hline & $\mathrm{Ag} / \mathrm{AgCl}$ & $10 / 10$ & & 97,76 & 96,59 \\
\hline
\end{tabular}

commercial electrodes are reasonably close, similarly to other studies in the field [30]. Remarkably, the result for dry electrodes is comparable to that of gelled $\mathrm{Ag} / \mathrm{AgCl}$ electrodes, meaning that the proposed electrolyte-free approach is able to deal with ECG signal acquisition even in dry conditions.

In order to evaluate the amount of baseline wandering artifact, it has been extracted from all the ECG recordings in the different conditions, as explained in Section II.C. An example with two different pairs of recordings on the same subject, with dry and wet textile electrodes, is presented in Fig. 9. The standard deviation of this narrowband component of the signal has been computed and synthetically represented in Fig. 10. Dry textile electrodes confirm the well-known baseline wandering problem, showing a larger dispersion of the standard deviation distribution both at rest and during exercise. Conversely, wet textile electrodes exhibit a similar behavior compared to the $\mathrm{Ag} / \mathrm{AgCl}$ ones.

Finally, the QRS detection analysis has been performed. The results are reported in Table II for dry textile electrodes and in Table III for wet textile electrodes in terms of number of readable signals, Sensitivity (S defined as $\mathrm{TP} /(\mathrm{TP}+\mathrm{FN})$ ) and Positive Predictivity $\left(\mathrm{P}^{+}=\mathrm{TP} /(\mathrm{TP}+\mathrm{FP})\right)$. The number of readable records is itself an index of the performance of the electrodes in terms of their ability in providing signals that could be used for heart rate analyses.

Since for the dry textile electrodes not all the signals acquired during exercises where readable, two sets of $\mathrm{S}_{\text {and }} \mathrm{P}^{+}$pairs have been considered to obtain a fair comparison between textile and $\mathrm{Ag} / \mathrm{AgCl}$ electrodes. The first one has been computed taking 
into account only the signals in which both textile and $\mathrm{Ag} / \mathrm{AgCl}$ electrodes produced readable traces, the latter considering the signals which were readable in at least one of the acquired ECG (in this case, only in $\mathrm{Ag} / \mathrm{AgCl}$ ones). In fact, when the signal is not readable, the results in terms of Sensitivity and Positive Predictivity are not meaningful, since the detection algorithm performs randomly. The results show the improvement due to wetting compared to dry condition, especially in terms of number of records in which it is possible to perform the QRS detection. Some subjects exhibit good quality signals even for dry textile electrodes. This is not always correlated to a low electrode-skin contact impedance, as could be figured out instead. The performance of dry textile electrodes are unsatisfactory in dynamic conditions, and acceptable at rest or during deep breathing, whereas wet textile electrodes show comparable or even better results than $\mathrm{Ag} / \mathrm{AgCl}$ ones, especially during exercise.

\section{DISCUSSION}

Taking into account the low-cost and simple fabrication process, and the fact that this is the first attempt to realize this kind of electrodes (to the best of our knowledge), these results are very encouraging. Some further analyses on the achieved results are provided hereafter.

A first consideration is related to a comparison with the electrodes presented in [24], which are based on a similar material and treatment. The conductivity of the fabrics achieved in our work is more than five times better than the conductivity reported by [24] with silk threads $(0.57 \mathrm{~S} / \mathrm{cm}$ vs $0.1 \mathrm{~S} / \mathrm{cm})$. Electrode-skin contact impedance values cannot be directly compared because in [24] they are not referred to humans, as in our case. Comparing our data with works that use the same type of material (polyester fabric) and treatment (PEDOT:PSS with sorbitol) but for other applications [40], our results are in any case in line with what is expected for the procedure $(0.22 \mathrm{~S} / \mathrm{cm}$ vs. $0.17 \mathrm{~S} / \mathrm{cm}$ for the sorbitol based treatment).

Even though biocompatibility studies should be carefully performed, biocompatibility of PEDOT:PSS electrodes is claimed in previous works in the literature [22], supporting the present application. Some issues could arise because of the potential presence of EDOT monomers, known to be toxic to cells, resisting the annealing process. Nevertheless, the use of a high-quality commercial dispersion ensures a low concentration of these free monomers. Furthermore, since PEDOT:PSS has shown to be thermally stable up to temperatures of $200^{\circ} \mathrm{C}$ [44], we can be quite confident that the annealing process does not disrupt the polymer structure nor produce potentially toxic byproducts.

Since the electrodes are handcrafted, the design is mainly dictated by the need for reproducibility and manufacturability. The size of the active area is comparable to that of other textile electrodes presented so far (e.g., $9 \mathrm{~cm}^{2}$ in [17], $10 \mathrm{~cm}^{2}$ in [16], up to $24 \mathrm{~cm}^{2}$ in [34]), even though someone presents a reduced area (e.g.: $4.5 \mathrm{~cm}^{2}$ in [3] up to $64 \mathrm{~mm}^{2}$ for some advanced materials). Compared to commercial electrodes, even though there are several smaller electrodes, some bigger ones also exist for diagnostic ECG (e.g., Covidien H49P are about $14.4 \mathrm{~cm}^{2}$ ) and others have comparable size (e.g., Covidien H59P are 8.4 $\mathrm{cm}^{2}$ ). In terms of full electrode size, the commercial ones used in this paper are far bigger, with a size of $43 \mathrm{~mm} \times 45 \mathrm{~mm}$. The size of the electrode has an obvious impact on the skin contact impedance, as occurs with the impedance of any other conductive path when the transverse area is changed. Reducing the active area will then lead to decreasing the quality of the recorded signal (attenuation, sensitivity to common mode noise, etc.). However, a detailed study on this aspect is out of the scope of this paper, mainly because of the manufacturing problems arising when the electrode size is reduced. Such studies have been performed on sewed electrodes in [15], revealing how small sizes affect the acquisition of the low-frequency components, but these results cannot be straightforwardly extended to the present work because of the different material.

All the measurements aiming at evaluating the effectiveness of the electrodes in sensing the electrophysiological signals have been carried out on human volunteers. Such a testbed is widely used in this field but, at the same time, it is questioned by some researchers, rising doubts on the reproducibility of the test scenarios. As a matter of fact, the properties of human skin differ from person to person and can change, for the same person, within hours [34] depending on the temperature and skin humidity, and also from region to region, reflecting the different skin composition [41]. Traditional metal-coated dry textile electrodes reveal a strong capacitive behavior mitigated by physiological (or artificial) skin wetting, resulting in a parallel conductive path [34]. Furthermore, the pressure exerted on the skin affects the conductivity [3] [29] [34], and the same holds for any electrode deformation (including stretch) due to the anatomical site of application.

For this reason, the indications provided by Fig. 4 and in the first box of Fig. 5 are more referable to the repeatability of the fabrication process and to general characteristics than to the precise assessment of the contact impedance on the human skin. This is clearly visible looking at the different dispersions in Fig. 5 for the dry electrodes (a condition in which the skin properties differences are exacerbated). Nevertheless, roughly speaking, these results compare well with other wearable electrodes in the literature. The impedance assumes a stable value after a period of 5 to 15 minutes, similarly to other textile electrodes [19]. They perform better than those in [18], which are not textile (polysiloxane loaded with conductive nano-particles), taking into account that such electrodes use a general-purpose electrolyte to provide the ion-conductivity. The results are also comparable with those in [19], related to textile electrodes made up of silver-coated polyester yarns. Such a similarity can be ascribed to the superficial characteristics of the textile electrodes, which depends on the weft, so that they could be improved by adopting non-woven supports [23].

In some impedance tests over 48 hours with our electrodes (data not shown), we observed that they progressively got dry within 3 hours (when wetted with two saline drops) and in 6 hours (when wetted with four saline drops). At that point, the impedance of the wet electrode becomes very similar to that of the dry one. Periodical wetting can be performed in some situations [12], keeping the impedance low. The electrodes can 
also be suited for recording during physical activities, since sweat has a positive impact on the performance of textile electrodes [38] and wet electrodes provided better performance than commercial ones when evaluated with the use of a QRS detector. Remarkably, none of the volunteers reported adverse effects or complaints about the test. Only the subject performing the 48 hours test reported temporary skin irritation and itch due to the belt (not to the electrodes). Furthermore, the volunteers could not perceive any substantial difference in terms of comfort when the electrode was wet, compared to the dry condition. This result could be exploited in some applications, as non-invasive fetal electrocardiography, where the high number of electrodes and the adhesive support cause substantial discomfort to the subject [45].

Results on ECG signal characteristics are also interesting, even though the population size is limited. The significance of such results is in any case limited because morphological analysis through in vivo comparison of different electrodes presents severe limitations. In fact, a non-identical positioning of the couples of electrodes for simultaneous recordings, as we did, can lead to differences in recorded signals that are not imputable to the electrodes [15]. Even putting the electrodes along the most probable cardiac axis direction can be cumbersome because of the variability of this parameter in different subjects [16]. Nevertheless, alternate measurements with different electrodes in the same position can erroneously lead to ascribe to the electrodes the morphological differences descending from the physio-pathological time variance of the ECG waveform [15].

In dry conditions, the proposed electrodes are able to operate correctly at rest, whereas during physical exercise artifacts heavily affect their behavior. This is due to the contact with the skin, which is not directly correlated to the active area of the electrode but rather to the skin deformation in that point [3] leading to an unstable skin electrode contact [16]. It should be also noted that, when used for the ECG recording, textile electrodes have been applied with a surgical TNT tape rather than with the elastic band, because of the anatomical site, so that the pressure on the skin is reduced and the electrode-skin contact impedance grows. All these problems can be mitigated by the adoption of a solid hydrogel membrane [30] [38], which is said to be critical for a correct acquisition in dynamic conditions. Remarkably, wet electrodes do not suffer this problem, leading to results that are comparable or better than those of $\mathrm{Ag} / \mathrm{AgCl}$ electrodes and also to active textile electrodes [17], even during dynamical tests. Being the wetting solution analog in composition to human sweat, it is predictable that, if the motion is intense enough to induce a small quantity of sweat, also dry electrodes can perform satisfactorily in dynamic conditions.

It is worth noting how the achieved results do not substitute an accurate clinical validation of the electrodes, which will be performed in the context of an approved clinical trial. In fact, if the aim is limited to the heart rate monitoring, the achieved results are sufficient. However, this is not true for ECG morphology analysis.

\section{CONCLUSION}

In conclusion, the comparison between the developed novel fully textile electrodes and the commercial ones has shown that their performances are comparable or even better in wet conditions (both at rest and in motion) while in dry conditions the results are comparable at rest but still worse in motion tests. On the other hand, textile electrodes, during motion, are beneficially affected by the production of sweat by the subject, thus indicating that they may be employed even in intense motion activities. The results shown in this paper actually demonstrate that the currently intended limits of textile electrodes may be overcome by both improving the quality of the treatment of textiles with conductive polymers (in contrast with what stated in [3]), and by a careful design of the electrode configuration (including suitable foam cushions aimed at keeping a constant pressure on the skin without compromising the comfort of the patient). Based on the promising results obtained on a significant number of healthy subjects, the future extension of this technique for the monitoring of larger sets of patients, as well as the application of these electrodes to the measurement of other kinds of biopotentials (e.g. EEG) will be soon considered.

Furthermore, the good performance demonstrated in shortterm monitoring of ECG paves the way towards the integration of the electrodes in smart garments for long-term monitoring. Work is in progress to investigate other aspects related to the integration in wearables as, for instance, washability and garment design.

\section{ACKNOWLEDGMENT}

The authors would like to thank Ms. Elisa Gusai for her collaboration to the experimental sessions, Dr. Ivan Spano for the support with the anechoic chamber setup, and all the colleagues who accepted to participate to the tests as voluntary subjects.

\section{REFERENCES}

[1] A. Lymberis, A. Dittmar, "Advanced Wearable Health Systems and Applications - Research and Development Efforts in the European Union," Engineering in Medicine and Biology Magazine, IEEE, vol. 26, no. 3, pp. 29-33, May-Jun. 2007.

[2] L. Su et al., "12-lead Holter electrocardiography," $J$ Herzschrittmachertherapie + Elektrophysiologie, vol. 24, n. 2, pp. 92-96, Jun. 2013.

[3] G. Cho et al., "Performance Evaluation of Textile-Based Electrodes and Motion Sensors for Smart Clothing," Sensors Journal, IEEE, vol.11, no. 12, pp. 3183-3193, Dec. 2011.

[4] A. Lymberis, S. Olsson, "Intelligent biomedical clothing for personal health and disease management: state of the art and future vision," Telemed. J. e-Health, vol. 9, no. 4, pp. 379-386, 2003.

[5] M. Mansoor Baig et al., "A comprehensive survey of wearable and wireless ECG monitoring systems for older adults," Med Biol Eng Comput, vol. 51, pp. 485-495, 2013.

[6] M. Di Rienzo et al., "Textile Technology for the Vital Signs Monitoring in Telemedicine and Extreme Environments," Information Technology in Biomedicine, IEEE Tran. on, vol. 14, no. 3, pp. 711-717, May 2010.

[7] J. Coosemans et al., "Integrating wireless ECG monitoring in textiles," Sensors and Actuators A: Physical, vol. 130-131, pp. 48-53, Aug. 2006.

[8] M. Di Rienzo et al., "Evaluation of a textile-based wearable system for the electrocardiogram monitoring in cardiac patients," Europace, vol. 15, pp. 607-612, 2013 .

[9] A. M. Bianchi et al., "Processing of signals recorded through smart devices: sleep-quality assessment," Trans. Info. Tech. Biomed., vol. 14, no. 3, pp. 741-747, May 2010. 
[10] M. Ishijima, "Unobtrusive approaches to monitoring vital signs at home," Medical \& Biological Engineering \& Computing, vol. 45, no. 11, pp. 1137-1141, Nov. 2007.

[11] G. M. Paul et al., "A Smart Textile Based Facial EMG and EOG Computer Interface," Sensors Journal, IEEE, vol. 14, no. 2, pp. 393-400, Feb. 2014.

[12] J. Löfhede et al., "Textile Electrodes for EEG Recording - A Pilot Study," Sensors, vol. 12, no. 12, pp. 16907-16919, 2012.

[13] C.-T. Lin et al., "Novel Dry Polymer Foam Electrodes for Long-Term EEG Measurement" Biomedical Engineering, IEEE Trans. on, vol.58, no.5, pp.1200-1207, May 2011.

[14] J. Crawford, L. Doherty, Practical Aspects of ECG Recording, M\&K Update Ltd, Aug. 2012.

[15] V. Marozas et al., "A comparison of conductive textile-based and silver/silver chloride gel electrodes in exercise electrocardiogram recordings," Journal of Electrocardiology, vol. 44, no. 2, pp. 189-194, 2011.

[16] T. Pola, J. Vanhala, "Textile Electrodes in ECG Measurement," in Proc. Intelligent Sensors, Sensor Networks and Information, ISSNIP. 3rd International Conference on, 2007, pp. 635-639.

[17] T.-H. Kang et al., "Nonwoven Fabric Active Electrodes for Biopotential Measurement During Normal Daily Activity," Biomedical Engineering, IEEE Trans. on, vol. 55, no. 1, pp. 188-195, Jan. 2008.

[18] K.-P. Hoffmann, R. Ruff, "Flexible dry surface-electrodes for ECG longterm monitoring," in Engineering in Medicine and Biology Society. EMBS 29th Annual International Conference of the IEEE, pp. 5739-5742, 2007.

[19] M. M. Puurtinen et al., "Measurement of noise and impedance of dry and wet textile electrodes, and textile electrodes with hydrogel," in Proc. Engineering in Medicine and Biology Society. EMBS 28th Annual International Conference of the IEEE, 2006, pp. 6012-6015.

[20] M. Stoppa, A. Chiolerio, "Wearable Electronics and Smart Textiles: A Critical Review," Sensors, vol. 14, pp. 11957-11992, 2014.

[21] G. Mattana et al., "Organic electronics on natural cotton fibres," Organic Electronics, vol. 12, no. 12, pp. 2033-2039, Dec. 2011.

[22] D. Khodagholy et al., "NeuroGrid: recording action potentials from the surface of the brain," Nature Neuroscience, vol. 18, pp. 310-315, 2015.

[23] T. I. Oh et al., "Nanofiber Web Textile Dry Electrodes for Long-Term Biopotential Recording," Biomedical Circuits and Systems, IEEE Trans. on, vol. 7, no. 2, pp. 204-211, Apr. 2013.

[24] S. Tsukada et al., "Conductive polymer combined silk fiber bundle for bioelectrical signal recording," PloS one, vol. 7, no. 4, p. e33689, Jan. 2012.

[25] Y. Chi, "Dry-contact and noncontact biopotential electrodes: Methodological review," Biomedical Engineering, IEEE Reviews in, vol. 3, pp. 106-119, 2010.

[26] R. Paradiso, M. Pacelli, "Textile electrodes and integrated smart textile for reliable biomonitoring," in Proc. 33rd Annual International Conference of the IEEE Engineering in Medicine and Biology Society, 2011, pp. 3274-3277.

[27] H. Okuzaki et al., "Highly conductive PEDOT/PSS microfibers fabricated by wet-spinning and dip-treatment in ethylene glycol," European Polymer Journal, vol. 45, no. 1, pp. 256-261, Jan. 2009.

[28] S. Kim, S. Leonhardt, "Influence of contact pressure and moisture on the signal quality of a newly developed textile ECG sensor shirt," in Proc. of the 5th International Workshop on Wearable and Implantable Body Sensor Networks, 2008, pp. 256-259.

[29] G. Paul et al., "The development of screen printed conductive networks on textiles for biopotential monitoring applications," Sensors and Actuators A: Physical, vol. 206, pp. 35-41, Feb. 2014.

[30] E. P. Scilingo et al., "Performance Evaluation of Sensing Fabrics for Monitoring Physiological and Biomechanical Variables" in IEEE Trans. on Information Technology in Biomedicine, vol. 9, no. 3, pp. 345-352, Sept. 2005.

[31] H. Carvalho et al., "Health monitoring using textile sensors and electrodes: An overview and integration of technologies," in Medical Measurements and Applications (MeMeA), IEEE International Symposium on, pp. 1-6, Jun. 2014.

[32] C. R. Merritt et al., "Fabric-Based Active Electrode Design and Fabrication for Health Monitoring Clothing," Information Technology in Biomedicine, IEEE Trans. on, vol. 13, no. 2, pp. 274-280, Mar. 2009.

[33] T. Vuorela et al., "Design and Implementation of a Portable Long-Term Physiological Signal Recorder," Information Technology in Biomedicine, IEEE Trans on, vol. 14, no. 3, pp. 718-725, May 2010.

[34] L. Beckman et al., "Characterization of textile electrodes and conductors," Physiol. Meas., vol. 31, pp. 233-247, 2010.
[35] G. D. Clifford, et al. "Signal quality indices and data fusion for determining clinical acceptability of electrocardiograms," Physiol. Meas., vol. 33, pp. 1419-1433, 2012.

[36] J. M. Łeskia, N. Henzel, "ECG baseline wander and powerline interference reduction using nonlinear filter bank," Signal Processing, vol. 85, no. 4, pp. 781-793, Apr. 2005.

[37] J. P. Martínez et al., "A Wavelet-Based ECG Delineator: Evaluation on Standard Databases," IEEE Trans. on Biomedical Engineering, vol. 51, no. 4, pp. 570-581, Apr. 2004.

[38] R. Paradiso et al., "A wearable health care system based on knitted integrated sensors," Information Technology in Biomedicine, IEEE Trans. on, vol. 9, no. 3, pp. 337-344, Sep. 2005.

[39] J. Pan, W. J. Tompkins, “A Real-Time QRS Detection Algorithm," IEEE Trans. on Biomedical Engineering, vol. BME-32, no. 3, pp. 230-236, Mar. 1985.

[40] Y. Ding et al., "Conductivity trends of PEDOT-PSS impregnated fabric and the effect of conductivity on electrochromic textile," ACS applied materials \& interfaces, vol. 2, no. 6, pp. 1588-1593, Jun. 2010.

[41] T. Sunaga et al., "Measurement of the electrical properties of human skin and the variation among subjects," Phys. Med. Biol., vol. 47, pp. N11, 2002.

[42] P. Kligfield et al, "Recommendations for the standardization and interpretation of the electrocardiogram: part I: the electrocardiogram and its technology: a scientific statement from the American Heart Association Electrocardiography and Arrhythmias Committee, Council on Clinical Cardiology; the American College of Cardiology Foundation; and the Heart Rhythm Society," Circulation, vol. 115, pp. 1306-1324, 2007.

[43] A. L. Goldberger et al., "PhysioBank, PhysioToolkit, and PhysioNet: Components of a New Research Resource for Complex Physiologic Signals," Circulation, vol. 101, no. 23, pp. e215-e220, Jun. 2000.

[44] S. Kirchmeyer et al., PEDOT: principles and applications of an intrinsically conductive polymer, New York, CRC Press, 2011, pp. 123125.

[45] D. Pani et al., "An advanced algorithm for fetal heart rate estimation from non-invasive low electrode density recordings," Physiological Measurements, vol. 35, no. 8, pp. 1621-1636, Aug. 2014 